\title{
Polarisierte Gesellschaft und polarisierte Kindheiten?
}

\author{
Die Differenzierung zwischen guten und schlechten Kindheiten wird stärker
}

In Deutschland wird die Kluft zwischen Arm und Reich seit Jahrzehnten größer. Nicht nur in finanzieller Hinsicht vergrößern sich die Abstände, auch räumlich leben arme und reiche Menschen nur noch selten Tür an Tür und begegnen sich kaum noch persönlich. Dies hat Konsequenzen auch für das gegenseitige Verständnis und das gesellschaftliche Vertrauen. Was bedeutet die Polarisierung der deutschen Gesellschaft für Kinder bzw. Kindheiten und wie wird das Aufwachsen durch diese Entwicklungen beeinflusst?

S eit Ende der 1970er-Jahre ist die Einkommensungleichheit in Deutschland kontinuierlich größer geworden. Dies zeigen neuere Berechnungen des Gini-Koeffizienten auf Basis der Einkommens- und Verbrauchsstichprobe (EVS). Auch das WSI kommt in der aktuellen Ausgabe seines Verteilungsberichtes (2019) zu dem Schluss, dass die Ungleichheit der verfügbaren Haushaltseinkommen seit knapp 30 Jahren größer geworden ist. Nach Daten des World Inequality Reports (WIR) und des Sozio-oekonomischen Panels (SOEP) ist die zunehmende Einkommensspreizung in Deutschland, wie auch in anderen Industrieländern, vor allem

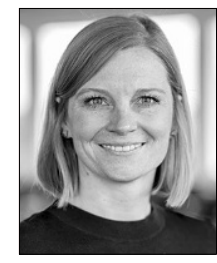

\section{Maren Hilke}

Technische Hochschule Köln, Köln, Deutschland

*1985; wissenschaftliche Mitarbeiterin an der Professur für kommunale Sozialpolitik der Technischen Hochschule Köln. maren.hilke@th-koeln.de

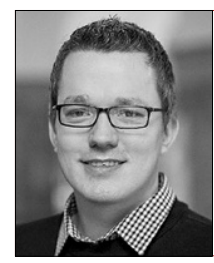

\section{Johannes Schütte}

Technische Hochschule Köln, Köln, Deutschland

*1982; Prof. Dr., Professor für Sozial- und Bildungspolitik an der Technischen Hochschule Köln.

johannes.d.schuette@gmail.com

Zusammenfassung Der Beitrag beschäftigt sich mit der Frage, wie die zunehmende gesellschaftliche Spaltung Kindheiten prägt. Dazu werden unterschiedliche Dimensionen gesellschaftlicher Polarisierung betrachtet. Neben der ökonomischen und der räumlichen Polarisierung rückt auch das Thema des gesellschaftlichen Zusammenhalts ins Zentrum der Betrachtung. Darauf aufbauend analysiert der Beitrag ungleiche Kindheiten unter dem Einfluss einer zunehmenden Polarisierung.

Schlüsselwörter Kinderarmut, Soziale Polarisierung, Kindheiten, Soziale Ungleichheiten auf Einkommenszugewinne am oberen Rand der Verteilung zurückzuführen (vgl. Hufe et al. 2018, S. 189; Spannagel und Molitor 2019).

Ökonomische, räumliche und soziale Polarisierung Wenn anstatt der tatsächlich verfügbaren Einkommen die Markteinkommen betrachtet werden, ist die Ungleichverteilung noch erheblich größer. Vor Steuern und Sozialleistungen liegt der Gini-Koeffizient für Deutschland anstatt bei rund 0,3 bei 0,4 . Schaut man sich die Entwicklung der Primär- und Sekundäreinkommen im Laufe der Zeit an, wird deutlich, dass es dem deutschen Sozialstaat nach wie vor gelingt, die primäre Verteilung der Markteinkommen so zu korrigieren, dass die Einkommenspolarisierung abgeschwächt wird. Gleichzeitig lässt sich dieser Dämpfungseffekt in den letzten Jahren immer weniger erkennen (Hufe et al. 2018, S. 189 ff.). Die Einkommenspolarisierung ist somit zunehmend staatlich mitverantwortet.

Die wachsende Ungleichheit zeigt sich nicht allein in der ,Fließgröße' Einkommen, sondern auch in der ,Bestandsgröße' des Wohlstands, also den Vermögen, die in Deutschland sogar noch weit ungleicher verteilt sind. So lag der Gini-Koeffizient für Vermögen im Jahr 2017 bei 0,78. Das reichste Zehntel der deutschen Bevölkerung verfügt über $56 \%$ des Gesamtvermögens. Im Vergleich dazu besitzen zwei Drittel der Bevölkerung kein oder ein sehr geringes Nettovermögen (vgl. Grabka und Halbmeier 2019). Bezogen auf die Entwicklung der Vermögensungleichheit kann bisher lediglich auf eine einzige aktuelle Studie zurückgegriffen werden. Die Erhebung von Albers et al. (2020) zeigt, dass sich die Vermögensverteilung in Deutschland seit der Wiedervereinigung stetig auseinanderentwickelt hat.

Auf Basis des bisher Gesagten erscheint es plausibel, dass die erkennbare ökonomische Polarisierung zu einer 
Zunahme der (Kinder-)Armut geführt hat. Dies bestätigt auch ein Blick auf die Entwicklung der Armuts(risiko) quote. So steigt nach Daten des Sozio-oekonomischen Panels (SOEP) der Anteil der Haushalte, die ein bedarfsgewichtetes Nettoeinkommen unter $60 \%$ des Medianeinkommens aufweisen, von rund $11 \%$ in den $1990 \mathrm{er}-$ Jahren auf aktuell 16,1\%. Die Werte für Kinder und Jugendliche liegen laut Mikrozensus und SOEP deutlich über der Quote von Erwachsenen, womit die Diagnose einer „Infantilisierung der Armut" von Richard Hauser (1997, S. 76) nach wie vor zutrifft.

Die Sozialstruktur der Gesellschaft spiegelt sich ebenfalls in der räumlichen Verteilung der Wohnstandorte. Residentielle Segregation entsteht durch unfreiwillige und freiwillige Entscheidungen und kann auch als sozialräumliche Polarisierung verstanden werden. Diese entsteht vor allem durch einen marktvermittelten Effekt: Wer in umkämpften Räumen über ökonomisches und soziales Kapital verfügt, kann seinen Wohnort wählen. Je kleiner das Kapitalvolumen, desto eher muss zum Wohnen gewählt werden, was der Markt übriglässt: Meist eine kleine Wohnung etwa in den Quartieren ehemaliger Industriestandorte, Großwohnsiedlungen oder an großen und lauten Durchgangsstraßen, mit einer Nachbarschaft, der der Markt ebenfalls keine Wahl ließ. Helbig und Jähnen (2018) stellen eine zunehmende Konzentration sozialer Benachteiligung in Nachbarschaften fest. In 36 der insgesamt 74 untersuchten deutschen Städten gibt es Quartiere „in denen der Anteil von Kindern mit SGB-II-Bezug bei über 50 Prozent liegt “" (vgl. ebd. S. 58). El-Mafaalani und Strohmeier (2015) beschreiben ebenfalls einen systematischen Zusammenhang zwischen sozialer (Konzentration von Armut) und demographischer Segregation (erhöhter Kinderanteil). Sie stellen fest, dass in den großen Städten der alten Bundesländer die Mehrheit der Kinder „in den ärmsten Stadtteilen mit hohen Ausländeranteilen [lebt], in denen die räumliche Konzentration benachteiligter und diskriminierter Menschen zusätzlich benachteiligend und diskriminierend wirkt" (ebd. S. 18).

Neben der Konzentration von Benachteiligung in Nachbarschaften kann auch in Institutionen wie Kindertageseinrichtungen (vgl. Groos und Jehles 2015) und Schulen (vgl. Helbig und Jähnen 2018) eine soziale Segregation beobachtet werden. Unterschiedliche Studien können eigenständige Effekte residentieller Segregation nachweisen. Die Ballung benachteiligter Kinder in Quartieren und Einrichtungen wirkt zusätzlich benachteiligend in Bezug auf die Gesundheit (vgl. u. a. El-Mafalaani und Strohmeier 2015), Bildung (vgl. Terpoorten 2014) und soziale Teilhabe (vgl. Friedrich und Blasius 2000). Diese Quartierseffekte ergeben sich aus unter- schiedlichen Begründungszusammenhängen, die sich unter drei Dimensionen (dem sozialen Milieu, der materiellen Ausstattung und dem Image) gruppieren lassen (vgl. Häußermann 2003). „Die räumliche Konzentration von Benachteiligung mündet also in benachteiligenden Quartieren, die Ausgrenzungsprozesse hervorrufen oder verstärken“ (ebd. S. 147) können.

Sowohl die dargelegte räumliche Polarisierung als auch die voranschreitende ökonomische Spaltung der Gesellschaft tragen dazu bei, dass sich die Lebenswelten und Aufwachsbedingungen der Kinder zunehmend unterscheiden. Familien haben aufgrund der größer werdenden Differenzen hinsichtlich der ihnen zur Verfügung stehenden finanziellen Mittel sehr unterschiedliche Herausforderungen zu bewältigen. So beschäftigen sich Gutverdienende vielleicht intensiv mit der Frage, wie sie eine sichere Altersvorsorge aufbauen können und ob es gewinnbringender ist, in Immobilien oder in Aktienfonds zu investieren. Beschäftigte im Niedriglohnsektor müssen hingegen von Tag zu Tag schauen, ob das Geld bis zum Ende des Monats reicht. Schlagen sich die Einen mit der Frage herum, welches Gemüse wirklich nachhaltig produziert wurde, fragen sich die Anderen, ob es heute bei der Tafel noch etwas Frisches geben wird. Diese Beispiele sollen deutlich machen, dass sich durch die ökonomischen Unterschiede auch die Wahrnehmungen verschieben können und damit auch die Vorstellungen, auf welchem Weg gesellschaftliche Probleme angegangen bzw. gelöst werden sollten. Illustrieren lässt sich dies ebenfalls mit einem geschichtlichen Zitat: Marie Antoinette soll zu einer Zeit, als das französische Volk hungerte, während die adeligen Eliten des Ancien Régime im Wohlstand lebten, gesagt haben: „Wenn sie kein Brot haben, dann sollen sie doch Kuchen essen! “. Dieses Zitat, das die Herrscherin nach heutigem Wissensstand zwar nie so gesagt hat, verdeutlicht eine vollkommene Distanzierung der Reichen von der Alltagswelt der armen Menschen. So kann es kaum verwundern, dass eine aktuelle Studie von Brand et al. (2020) zu dem Ergebnis kommt, dass die gesellschaftliche Gerechtigkeit in Abhängigkeit von der sozialen Stellung bewertet wird und auch die Bereitschaft sich zivilgesellschaftlich abnimmt. Brand et al. (2020) resümieren, „dass Armut und prekäre Lebenslagen sowohl individuell als auch kollektiv die größte Bürde für den Zusammenhalt sind. Wer sich hauptsächlich um das eigene Auskommen Sorgen machen muss, dem fehlt die Zeit und möglicherweise auch das Interesse, sich für das Gemeinwesen einzusetzen. Wenn nennenswerte Teile der Gesellschaft das Gefühl haben, ihre Interessen würden nicht berücksichtigt und es gehe nicht gerecht zu, dann belastet das auch das politische Vertrauen“ (ebd. S. 77 f). 
Zusätzlich begegnen sich Menschen aus unterschiedlichen Sozialschichten aufgrund der räumlichen Segregation immer weniger. Armut erscheint für Menschen in bestimmten wohlhabenden Vierteln als etwas sehr Theoretisches was sie nicht mehr aus ihrem eigenen Erleben, sondern vielleicht noch aus Beschreibungen in Romanen, der Zeitung oder dem Fernsehen kennen. Und auch für Kinder, die in der KiTa oder Schule keinen Kontakt zu armen Kindern haben, ist es unvorstellbar, dass Familien nicht in großzügigen Häusern mit Garten leben und jedes Jahr mehrfach verreisen. Dazu kommt noch, dass sowohl die Qualität als auch die Quantität sozialer Kontakte insgesamt von der gesellschaftlichen Stellung abhängig sind. So zeigen Böhnke und Link (2018), dass einkommensarme Personen im Durchschnitt weniger sozial vernetzt sind als nicht einkommensarme Personen und es mit zunehmender Armutsdauer zu einer schichtspezifischen Homogenisierung der Sozialkontakte kommt.

Ökonomische und räumliche Polarisierung gipfeln somit in einer sozialen Polarisierung der Gesellschaft. Die bereits erwähnte Studie von Brand et al. (2020) findet Hinweise auf einen abnehmenden gesellschaftlichen $\mathrm{Zu}$ sammenhalt in Deutschland. Die Autor_innen machen dies vor allem an der wachsenden regionalen Spreizung bei der Akzeptanz von Diversität fest (vgl. ebd. S. 54 ff). Dieser Trend passt zu dem Befund, dass die zunehmenden Wahlerfolge populistischer und teils rechtsradikaler Parteien eine beträchtliche Wahlunterstützung von Wähler_innen erhalten, die am meisten unter den gesellschaftlichen Ungleichheiten zu leiden haben, nämlich von Menschen aus niedrigen gesellschaftlichen Schichten (vgl. Proaño et al. 2020).

\section{Polarisierte Kindheiten}

Die bisherige Darstellung hat gezeigt, dass in unterschiedlichen Dimensionen gesellschaftliche Polarisierungsentwicklungen zu beobachten sind, die in einem engen Verweisungszusammenhang stehen. Einhergehend mit dieser Entwicklung hat die Anzahl der Kin- der, die von relativer Armut betroffen sind, seit Jahren zugenommen. Darüber hinaus lässt die vorhandene empirische Datenlage einen Zusammenhang zwischen (relativer) Kinderarmut und negativen Entwicklungs- und Teilhabechancen als evident erscheinen. Dies belegen verschiedene empirische Studien (vgl. z. B. Tophoven et al. 2018), in denen die komplexen Folgen von Kinderarmut in unterschiedlichen Dimensionen aufgezeigt werden. Bezogen auf den Lebensraum von Kindern zeigt sich dies beispielsweise bei der Belastung mit gesundheitsschädlichen Staub-, Fluor-, Schwefeldioxid-Immissionen, den Lärmbelastungen am Wohnort und bei der Größe und Qualität der Wohnungen (vgl. Mielck und Heinrich 2002).

Kinderarmut hat ebenfalls Auswirkungen auf die Gesundheit der Kinder. Kinder und Jugendliche aus $\mathrm{Fa}$ milien mit einem niedrigen gesellschaftlichen Status ernähren sich durchschnittlich ungesünder, sind weniger körperlich aktiv, besitzen ein höheres Risiko, psychische Erkrankungen oder Verhaltensauffälligkeiten zu entwickeln und haben ein höheres Risiko, an Übergewicht und Adipositas zu erkranken (vgl. RKI und BZGA 2008) im Vergleich zu nicht armen Kindern. Darüber hinaus zeigt sich ein Zusammenhang zwischen Kinderarmut und den Chancen im Bildungsbereich. Kinder aus Familien in Risikolagen (niedriges Einkommen, niedriger Bildungsstand, geringe soziale Einbindung), haben schlechtere Chancen, das Abitur zu machen, als Kinder aus Familien ohne Risikolagen (vgl. Autorengruppe Bildungsberichterstattung 2018, S. 35 ff.). Die beschriebenen Ergebnisse zeigen, dass sich Kindheiten schichtspezifisch unterscheiden und man ungleiche Kindheiten in Bezug auf eine ungleiche Verteilung von Ressourcen und ungleichen Chancen zur Teilhabe an Gesellschaft beobachten kann. Aus dieser Perspektive kann somit von einer Polarisierung der Teilhabechancen von Kindern gesprochen werden.

Neben der wachsenden Ungleichheitsproblematik, die maßgeblich auf die Ressourcenverteilung und damit auf gesellschaftliche Machtverhältnisse zurückgeführt wer-

Hier steht eine Anzeige. 
den kann, lassen sich normative Prägungen und Bewertungen von Kindheiten beobachten. Diese zunehmende Bewertung von Kindheiten anhand dominierender gesellschaftlicher Vorstellungen führt zu einer stärker werdenden Differenzierung zwischen guten und schlechten Kindheiten. Die momentan vorherrschenden Vorstellungen einer guten Kindheit oder auch gelingenden Aufwachsens sind geprägt durch normative Muster einer „behüteten Kindheit“ (vgl. Bühler-Niederberger 2011, S. $41 \mathrm{ff}$.). Diese Idealvorstellung ist verknüpft mit einem traditionellen Bild einer Normalfamilie. Dieses Familienbild beinhaltet neben dem männlichen Ernährermodell einer heterosexuellen Ehe mit ein bis zwei Kindern auch eine starke Mittelschichtsorientierung. Familienkonstellationen wie z. B. die arbeitslose Alleinerziehende mit drei Kindern werden kategorisch als defizitär gerahmt und institutionell abgewertet. Dies zeigt sich z. B. an der Gestaltung institutioneller Arrangements wie dem Ehegattensplitting oder auch in Konzepten und Strukturen pädagogischer Einrichtungen.

Mit solchen institutionellen Diskriminierungen (Radke und Gomolla 2009) bestimmter nicht normgerechter Familienkonstellationen, geht eine Abwertung von Kindheiten einher, die nicht in diesen Verhältnissen stattfinden. Im Schulsystem beispielsweise werden „Eigenschaften und Ergebnisse der Mittelschichtskindheiten höher bewertet als die anderer Kindheiten - auch unabhängig von der durch die Kinder messbar erzielten kognitiven Leistungsfähigkeit" (Bühler-Niederberger und Mierendorff 2009, S. 451). So zeigt sich, dass schulische Leistungsbewertungen davon abhängig sind, ob die Freizeitgestaltung der Kinder den Vorstellungen der Lehrerinnen und Lehrer entspricht, oder eben nicht (vgl. Furtner-Kallmünzer et al. 2002, S. 253 ff.). Soziale Herkunft und Lebenslage der Kinder und deren Passung an institutionelle Erwartungen haben Einfluss auf die Teilhabechancen. Angefangen von einer schichtspezifischen Praxis der Notengebung, über eine ebenfalls schichtspezifisch beeinflusste Schulwahlentscheidung bis hin zu unterschiedlichen Einflüssen auf die Herausbildung von im Schulkontext positiv bewerteten Kompetenzen, werden im Fachdiskurs sehr unterschiedliche Ursachenzusammenhänge diskutiert. Über institutionelle Bewertungsprozesse findet so eine implizite Diskriminierung statt und die normative Vorstellung einer behüteten Kindheit erscheint als etwas von Natur aus Gutes. Durch eine solche Naturalisierung werden die Herrschaftsstrukturen verschleiert und als naturgegeben legitimiert. Aus Unterschieden werden Unterscheidungen (Distinktionen). Die Naturalisierung sozialer Unterschiede trägt dazu bei, dass selbst die Benachteiligten die vermeintliche Überlegenheit der Herrschen- den anerkennen. Aufgrund der Allgegenwärtigkeit der Normvorstellung einer behüteten Kindheit und da diese weitestgehend als unhinterfragter Konsens gilt, bewerten Menschen aus den unteren Sozialschichten ihre eigenen Vorstellungen von Kindheit mehr und mehr als defizitär. Diese Mechanismen diskutiert Bourdieu unter dem Begriff symbolische Gewalt. Sie wirkt als verdeckte Art der Herrschaftsausübung, die nicht als solche wahrgenommen wird und die Zustimmung der Beherrschten findet.

Der systematische Ausbau der Hilfe- und Betreuungsangebote für Kinder in den letzten Jahren hat dazu geführt, dass (gerade kleine) Kinder mehr denn je unter institutioneller Beobachtung stehen. Kinder besuchen früher und länger eine institutionelle Betreuung, darin wird ihre Entwicklung beobachtet und dokumentiert. Präventiv ausgerichtete Angebote wie die Frühen Hilfen und Vorsorgeuntersuchungen von Kindern, die einer frühzeitigen Erkennung von körperlichen, geistigen und sozialen Entwicklungsdefiziten $(\mathbb{S} 26$ SGB V) dienen, werden ausgebaut und die Bereitschaft zur Teilnahme vor allem von Risikogruppen eingefordert, um nur einige Beispiele zu nennen. Aus diesem Trend zur stärkeren Beobachtung resultieren auch häufigere Diagnosen von Fehlentwicklungen bzw. Normabweichungen kindlicher Entwicklung und damit auch Korrekturbemühungen. Hier wird deutlich, dass eine Orientierung an einem normativen Muster kindlicher Entwicklungen erst Abweichungen und Ungleichheiten sichtbar und problematisierbar macht (vgl. Bühler-Niederberger und Mierendorff 2009, S. 453).

Außerdem ist, besonders in den Diskussionen um Kinderschutz und Prävention, ein zunehmendes Misstrauen gegenüber armen Eltern erkennbar. So wird diesen Eltern nicht zugetraut, dass sie ihren Kindern die als notwendig beschriebenen Entwicklungsbedingungen bieten und sie normgerecht erziehen. Dieses Misstrauen zeigt sich unter anderem im öffentlichen Diskurs zu häuslichen Lernumgebungen und elterlichen Kompetenzen (vgl. Betz et al. 2017). Durch Defizitzuschreibungen wie z. B. „Risikomütter“ werden Armutsfolgen vorrangig als individuell-moralisches Problem gerahmt und die öffentliche Diskreditierung armer Eltern führt dazu, dass das Verhalten armer Eltern in den Mittelpunkt rückt, nicht die strukturellen Ursachen. Die Verantwortung für das institutionelle Scheitern armer Kinder wird auf diese Weise individualisiert. Eine solche Responsibilisierung rechtfertigt wiederum eine noch stärkere Überwachung von Kindern und ein frühes disziplinierendes Eingreifen durch öffentliche Institutionen.

Gleichzeitig sind Muster einer „guten Kindheit“ immer anspruchsvoller geworden. Eltern aus mittleren Schich- 
ten investieren zunehmend ,in die frühe Akkumulation von bildungsrelevantem Kulturkapital ihrer Kinder“" (Bühler-Niederberger und Mierendorff 2009, S. 452). Betrachtet man beispielsweise die Betreuung der unter Dreijährigen in Kindertageseinrichtungen zeigt sich, dass insbesondere Eltern der Mittelschicht ihre Kinder früh und lange in eine Betreuung geben. Gerade durch die Angebotsknappheit der Plätze für Kinder unter drei Jahren werden Familien mit schlechteren ökonomischen und sozialen Ressourcen verdrängt und ihnen der $\mathrm{Zu}$ gang erschwert (vgl. Baader et al. 2011). Neben der institutionellen frühkindlichen Bildung und Betreuung wird auch ein Unterschied in der Nutzung von Angeboten der Frühen Hilfen deutlich. Insbesondere bei Kursund Gruppenangeboten, deren Anspruch und Ziel es ist, Eltern in ihren Erziehungskompetenzen zu stärken und für die Bedürfnisse ihres Kindes zu sensibilisieren, zeigt sich, dass diese häufiger von Mittelschichtsfamilien frequentiert werden (vgl. Franzke und Schultz 2016). Eine zunehmende Investition von Mittelschichtsfamilien in bildungsrelevantes Kulturkapital ihrer Kinder zeigt sich auch später in der Biographie beispielsweise im Besuch von Privatschulen. Zahlen des Statistischen Bundeamtes zeigen, dass die Zahl der Privatschulen von 1992 bis 2017 von 3232 auf 5839 gestiegen ist (vgl. Rudnicka 2020).

\section{Fazit}

Zusammenfassend lässt sich festhalten, dass Deutschland nicht nur eine sich zunehmend polarisierende Gesellschaft darstellt, sondern sich hierzulande auch Kindheiten polarisieren. Es konnte gezeigt werden, dass sich Polarisierungstendenzen in der ökonomischen, räumlichen und sozialen Dimension abzeichnen und diese in engen Wechselbeziehungen zu einander stehen. Kindheiten finden innerhalb von Gesellschaften statt, prägen diese und werden davon geprägt. Eine zunehmende Polarisierung von Kindheiten lässt sich aus zwei Begründungszusammenhängen herleiten, die in Verbindung miteinander zu sehen sind: Die wachsende soziale Ungleichheit zeigt sich insbesondere für die Gruppe der Kinder. Armut hat komplexe Folgen für kindliche Teilhabechancen. Damit sind Kindheiten polarisiert zwischen armen und reichen Kindheiten. Eine Polarisierung von Kindheiten kann darüber hinaus mit einer zunehmenden normativen Prägung und Bewertung der Lebensphase Kindheit begründet werden. Diese zunehmende Bewertung von Kindheiten anhand dominierender gesellschaftlicher Vorstellungen führt zu einer stär- ker werdenden Differenzierung zwischen guten und schlechten Kindheiten.

Eingegangen. 11. September 2020

Angenommen. 28. September 2020

Funding. Open Access funding enabled and organized by Projekt DEAL.

Open Access. Dieser Artikel wird unter der Creative Commons Namensnennung 4.0 International Lizenz veröffentlicht, welche die Nutzung, Vervielfältigung, Bearbeitung, Verbreitung und Wiedergabe in jeglichem Medium und Format erlaubt, sofern Sie den/die ursprünglichen Autor(en) und die Quelle ordnungsgemäß nennen, einen Link zur Creative Commons Lizenz beifügen und angeben, $\mathrm{ob}$ Änderungen vorgenommen wurden.

Die in diesem Artikel enthaltenen Bilder und sonstiges Drittmaterial unterliegen ebenfalls der genannten Creative Commons Lizenz, sofern sich aus der Abbildungslegende nichts anderes ergibt. Sofern das betreffende Material nicht unter der genannten Creative Commons Lizenz steht und die betreffende Handlung nicht nach gesetzlichen Vorschriften erlaubt ist, ist für die oben aufgeführten Weiterverwendungen des Materials die Einwilligung des jeweiligen Rechteinhabers einzuholen.

Weitere Details zur Lizenz entnehmen Sie bitte der Lizenzinformation auf http://creativecommons.org/licenses/by/4.0/deed.de

\section{Literatur}

Albers, T., Bartels, C., \& Schularick, M. (2020). The Distribution of Wealth in Germany, 1895-2018. In ECONtribute Working Paper, 2020/1. https://selten.institute/wp-content/uploads/2020/03/ECONtribute_The_Distribution_of_Wealth_eng_study.pdf. Zugegriffen: 28. Sept. 2020.

Autorengruppe Bildungsberichterstattung (2018). Ein indikatorengestützter Bericht mit einer Analyse zu Wirkungen und Erträgen von Bildung. Bildung in Deutschland 2018. Bielefeld: wbv.

Baader, M. S. (2011). Frühkindliche Bildung, Betreuung und Erziehung aus der Perspektive sozialer Ungleichheit. In Hans-Böckler-Stiftung (Hrsg.), Arbeitspapier 197. Düsseldorf: Hans-Böckler-Stiftung. https://www.boeckler.de/pdf/p_arbp_197.pdf. Zugegriffen: 28. Sept. 2020.

Betz, T., Honig, M., \& Ostner, I. (2017). Parents in the spotlight. Parenting practices and support from a comparative perspective. Sonderheft der Zeitschrift für Familienforschung, Bd. 11. Leverkusen: Barbara Budrich.

Böhnke, P., \& Link, S. (2018). Armut, soziale Netzwerke und Partizipation. In: P. Böhnke, J. Dittmann \& J. Goebel (Hrsg.), Handbuch Armut. Ursachen, Trends, Maßnahmen (S. 247-257). Stuttgart: utb.

Brand, T., Follmer, R., \& Unzicker, K. (2020). Gesellschaftlicher Zusammenhalt in Deutschland 2020. Eine Herausforderung für uns alle. Ergebnisse einer repräsentativen Bevölkerungsstudie. Gütersloh: infas - Institut für angewandte Sozialwissenschaft GmbH und Bertelsmann Stiftung.

Bühler-Niederberger, D. (2011). Lebensphase Kindheit. Theoretische Ansätze, Akteure und Handlungsräume. Weinheim, München: Juventa.

Bühler-Niederberger, \& Mierendorff, D. J. (2009). Ungleiche Kindheiten eine kindheitssoziologische Annäherung. In Diskurs Kindheits- und Jugendforschung (Bd. 4, S. 449-456).

El-Mafalaani, A., \& Strohmeier, K. P. (2015). Segregation und Lebenswelt. Die räumliche Dimension sozialer Ungleichheit. In A. El-Mafalaani, S. Kurtenbach \& K. P. Strohmeier (Hrsg.), Auf die Adresse kommt es an. Segregierte Stadtteile als Problem- und Möglichkeitsräume begreifen (S. 18-42). Weinheim, Basel: Beltz Juventa. 


\section{Extrablick: (Kinder-)Armut}

Franzke, A., \& Schultz, A. (2016). Früh übt sich... Bedingungen und Formen der Inanspruchnahme präventiver Angebote von Familien mit dreijährigen Kindern. In Bertelsmann Stiftung (Hrsg.), Bd. 5. Gütersloh: Bertelsmann Stiftung. https://www.bertelsmann-stiftung.de/fileadmin/files/BSt/ Publikationen/GrauePublikationen/Werkstattbericht_Bd._5_Orange_R_ KeKiz_Frueh_uebt_sich.pdf. Zugegriffen: 23. Sept. 2020.

Friedrichs, J., \& Blasius, J. (2000). Leben in benachteiligten Wohngebieten. Opladen: Budrich.

Furtner-Kallmünzer, et al. (2002). In der Freizeit für das Leben lernen. Eine Studie zu den Interessen von Schulkindern. München: Deutsches Jugendinstitut.

Grabka, M., \& Halbmeier, C. (2019). Vermögensungleichheit in Deutschland bleibt trotz deutlich steigender Nettovermögen anhaltend hoch. DIW Wochenbericht, 86(40), 735-745.

Groos, T., \& Jehles, N. (2015). Der Einfluss von Armut auf die Entwicklung von Kindern. Ergebnisse der Schuleingangsuntersuchung. Gütersloh: Bertelsmann Stiftung.

Hauser, R. (1997). Vergleichende Analyse der Einkommensverteilung und der Einkommensarmut in den alten und neuen Bundesländern 1990 bis 1995. In I. Becker \& R. Hauser (Hrsg.), Einkommensverteilung und Armut. Deutschland auf dem Weg zur Vierfünftel-Gesellschaft? (S. 63-82). Frankfurt a.M., New York: Campus.

Häußermann, H. (2003). Armut in der Großstadt. Die Stadtstruktur verstärkt soziale Ungleichheit. Information zur Raumentwicklung, 3/ 4, 147159.

Helbig, M., \& Jähnen, S. (2018). Wie brüchig ist die soziale Architektur unserer Städte? Trends und Analysen der Segregation in 74 deutschen Städten. Discussion Paper P 2018-001. Berlin: Wissenschaftszentrum Berlin für Sozialforschung. https://bibliothek.wzb.eu/pdf/2018/p18-001.pdf. Zugegriffen: 25. Sept. 2020

Hufe, P., Peichl, A., \& Stöckli, M. (2018). Ökonomische Ungleichheit in Deutschland - ein Überblick. Perspektiven der Wirtschaftspolitik, 19(3), 185-199.

Mielck, A., \& Heinrich, J. (2002). Soziale Ungleichheit und die Verteilung umweltbezogener Expositionen, Environmental Justice. Das Gesundheitswesen, 64(7), 405-416.

Proaño, C. R., Peña, J. C., \& Saalfeld, T. (2020). Inequality, macroeconomic performance and political polarization: a panel analysis of 20 advanced democracies. Working Paper No. 157 June 2020. https://www.uni-bamberg.de/fileadmin/uni/fakultaeten/sowi_faecher/vwl/BERG/BERG_149.pdf. Zugegriffen: 27. Sept. 2020.

Radtke, F. O., \& Gomolla, M. (2009). Institutionelle Diskriminierung. Die Herstellung ethnischer Differenz in der Schule. Wiesbaden: VS Verlag für Sozialwissenschaften.

Robert Koch-Institut (RKI), \& Bundeszentrale für gesundheitliche Aufklärung (Hrsg.). (2008). Erkennen - Bewerten - Handeln: Zur Gesundheit von Kindern und Jugendlichen in Deutschland. Berlin: RKI.

Rudnicka, J. (2020). Private Schulen in Deutschland bis 2018. https:// de.statista.com/statistik/daten/studie/954564/umfrage/private-schulen-indeutschland/. Zugegriffen: 25. Sept. 2020.

Spannagel, D., \& Molitor, K. (2019). WSI-Verteilungsbericht 2019. Einkommen immer ungleicher verteilt. Report (53). Düsseldorf: WSI.

Terpoorten, T. (2014). Räumliche Konfiguration der Bildungschancen. Segregation und Bildungsdisparitäten am Übergang in die weiterführenden Schulen im Agglomerationsraum Ruhrgebiet. ZE-

FIR-Materialien 3. Bochum. http://www.zefir.rub.de/mam/content/ zefir_materialien_3_r\% C3\% A4umliche_konfiguration_der_bildungschancen_2014.pdf. Zugegriffen: 28. Sept. 2020.

Tophoven, S., Lietzmann, T., Reiter, S., \& Wenzig, C. (2018). Aufwachsen in Armutslagen. Zentrale Einflussfaktoren und Folgen für die soziale Teilhabe. Studie vom Institut für Arbeitsmarkt- und Berufsforschung (IAB) im Auftrag der Bertelsmann Stiftung. Gütersloh: Bertelsmann.

Hier steht eine Anzeige. Springer 